\title{
Exact Performance Analysis of Optimum Combining With Multiple Interferers in Flat Rayleigh Fading
}

\author{
Peter J. Smith
}

\begin{abstract}
This letter provides a comprehensive overview and extension of recent results on outage probabilities and bit-error rates (BER) for optimal combiners in the presence of multiple interferers and additive noise. Desired signal and interferers are subject to flat Rayleigh fading and all channels are independent. In addition to summarizing previous work, this letter also derives the BER for a wider range of modulations than previously considered. We show that previous approximate results on the equal power interferer case where the number of interferers is less than the number of antenna elements can be made exact in a straightforward way. Finally we extend previous work on the single and double interferer case to the general case of arbitrary numbers of interferers.
\end{abstract}

Index Terms-MMSE linear combining, optimum combining, rayleigh fading.

\section{INTRODUCTION}

W E CONSIDER the performance of ideal minimum mean-square error (MMSE) combiners using linear combining in the presence of multiple interferers with Rayleigh fading additive interference channels. Such systems have been studied intensively [1]-[9] and it is well known that the MMSE solution corresponds to maximizing the output signalto-interference-plus-noise ratio (SINR). The most complete analysis to date is that given in [10]-[12] which provides exact formulations for the density of the output SINR and outage probabilities. As a special case of this work, it is straightforward to consider the situation where the interferers have equal powers and there are fewer interferers than antenna elements. Hence, the approximate densities and outage probabilities discussed in [13] can be made exact. In [13] approximate BER values are also derived for a range of modulations. In situations where the conditional probability of error is an exponential function of the SINR (i.e., DPSK) the exact BER is given in [14], [15] for the general case. For other modulations, i.e., BPSK or OFSK, the conditional probability of error contains the complementary error function. Here fewer results are available. In [16], the exact BER is given for the single interferer case in a form which requires numerical integration. In [17], the case of two interferers is handled exactly. In Section III-B we extend these results to give the solution for an arbitrary number of interferers without the need for numerical integration. In summary, we derive the exact BER for all three modulations for an arbitrary number of

Paper approved by V. A. Aalo, the Editor for Transmission Systems of IEEE Communications Society. Manuscript received August 7, 2001; revised December 2, 2002.

The author is with the Department of Electrical and Electronic Engineering, University of Canterbury, Christchurch 8140, New Zealand (e-mail: p.smith@elec.canterbury.ac.nz).

Digital Object Identifier 10.1109/TCOMM.2007.904354 interferers. For completeness we also give the exact BER results for the special case discussed in [13].

The layout of the letter is as follows. In Section II, we recall exact formulae for the general output SINR distribution [10]-[12] and derive equivalent results for the equal power interferer special case [13]. In Section III we quote some previous BER results and derive the exact BER for some new modulation types. Results are given both for the general case and the equal power interferer case [13].

\section{THE SINR DISTRIBUTION}

The context is identical to that considered in [12] where $M$ antenna elements are used in linear MMSE combining with $N$ interferers in a Rayleigh fading channel with additive noise. It is assumed that the desired and interfering sources are from some iid zero-mean random process with magnitude variance $a^{2}$. The average received power of the $n$th source is $a^{2} P_{n}$ at each antenna element where $n=0$ denotes the desired source and $n \in\{1,2, \ldots, N\}$ denotes the interferers. The relative interferer powers are denoted $\Gamma_{n}=P_{n} / P_{0}$. The additive noise is modeled by an independent zero-mean complex Gaussian noise process with magnitude variance $\sigma^{2}$. The average signal-to-noise ratio at each combiner input is denoted by $\gamma=a^{2} P_{0} / \sigma^{2}$.

If $Z$ is the combiner's output SINR for such a system then $F(z)=P(Z \leq z)$ is the outage probability and $R(z)=1-F(z)$ is the reliability. In [12] the reliability function is derived as

$$
R(z)=\exp (-z / \gamma) \sum_{m=1}^{M} \frac{A_{m}(z)}{(m-1) !}(z / \gamma)^{m-1}
$$

where

$$
A_{m}(z)= \begin{cases}1, & M \geq N+m \\ \frac{1+\sum_{i=1}^{M-m} C_{i} z^{i}}{\prod_{n=1}^{N}\left(1+\Gamma_{n} z\right)}, & M<N+m\end{cases}
$$

and $C_{i}$ is the coefficient of $z^{i}$ in $\prod_{n=1}^{N}\left(1+\Gamma_{n} z\right)$, i.e.,

$$
C_{i}=\sum_{1 \leq n_{1}<\cdots<n_{i} \leq N} \Gamma_{n_{1}} \Gamma_{n_{2}} \cdots \Gamma_{n_{i}} .
$$

In fact, computation of $A_{m}(z)$ is best performed by the simple recursion in [12] rather than the direct use of (2) and (3). The SINR density is also given in [12] for the interference limited case $\left(\sigma^{2}=0\right)$ and has the simple form

$$
f(z)=\sum_{i=1}^{N} \alpha_{i}\left(1+\Gamma_{i} z\right)^{-2}
$$


where

$$
\begin{aligned}
\alpha_{i}= & (-1)^{N-M} \Gamma_{i}^{N-M+1} \sum \Gamma_{i_{1}} \Gamma_{i_{2}} \cdots \Gamma_{i_{M-1}} \\
& \times\left[\prod_{j \neq i}\left(\Gamma_{j}-\Gamma_{i}\right)\right]^{-1}
\end{aligned}
$$

and the summation in (5) is over all $1 \leq i_{1}<i_{1}<\cdots<$ $i_{M-1} \leq N$ such that $i_{j} \neq i$.

For large values of $M, N(5)$ can be numerically difficult and so (4) is rewritten in [14] to give the numerically stable version

$$
f(z)=\sum_{i=1}^{N} d_{i} z^{M+i-2} \prod_{j=1}^{N}\left(1+\Gamma_{j} z\right)^{-2}
$$

where

$$
d_{i}=\sum_{k=\max (0, i-N+M+1)}^{\min (M-1, i-1)}(i-2 k+M-1) C_{k} C_{i-k+M-1}
$$

and $C_{i}$ is defined in (3). Versions of (4) and (6) for the case where $\sigma^{2} \neq 0$ can also be found in [14].

Now consider the special case discussed in [13] where there are more antenna elements than interferers. If we let $M=N+$ $B$ then [12] gives the version of (1), as shown below:

$$
\begin{aligned}
R(z)= & \exp (-z / \gamma) \sum_{m=1}^{B} \frac{(z / \gamma)^{m-1}}{(m-1) !} \\
& +\exp (-z / \gamma) \sum_{m=B+1}^{M} \frac{(z / \gamma)^{m-1}}{(m-1) !} A_{m}(z)
\end{aligned}
$$

For the equal interferer power case we have $\Gamma_{1}=\Gamma_{2}=\cdots=$ $\Gamma_{N}=\Gamma$ and (8) collapses to

$$
\begin{aligned}
R(z)= & \exp (-z / \gamma) \sum_{m=1}^{B} \frac{(z / \gamma)^{m-1}}{(m-1) !}+\exp (-z / \gamma) \\
& \times \sum_{m=B+1}^{M} \frac{(z / \gamma)^{m-1}}{(m-1) !} \sum_{i=0}^{M-m}\left(\begin{array}{c}
N \\
i
\end{array}\right)(\Gamma z)^{i}(1+\Gamma z)^{-N}(9)
\end{aligned}
$$

Writing (9) as $R(z)=R_{1}(z)+\exp (-z / \gamma) R_{2}(z)$ we see that the density can be written as

$$
\begin{aligned}
f(z) & =-R_{1}^{\prime}(z)+\gamma^{-1} \exp (-z / \gamma) R_{2}(z)-\exp (-z / \gamma) R_{2}^{\prime}(z) \\
& =-R_{1}^{\prime}(z)+R_{3}(z)
\end{aligned}
$$

Some straightforward differentiation gives

$R_{1}^{\prime}(z)=\gamma^{-1} \exp (-z / \gamma) \sum_{m=1}^{B} \frac{(z / \gamma)^{m-2}}{(m-1) !}[m-1-z / \gamma]$

and

$$
\begin{aligned}
R_{3}(z)= & \exp (-z / \gamma) \sum_{m=B+1}^{M}\left\{\left(\frac{1}{\gamma}-\frac{m-1}{z}+\frac{N \Gamma}{1+\Gamma z}\right) t_{m}(z)\right. \\
& \left.-\frac{(z-\gamma)^{m-1}}{(m-1) !}\left[\sum_{i=1}^{M-m}\left(\begin{array}{c}
N \\
i
\end{array}\right)(i \Gamma)(\Gamma z)^{i-1}(1+\Gamma z)^{-N}\right]\right\}
\end{aligned}
$$

where

$$
t_{m}(z)=\frac{(z / \gamma)^{m-1}}{(m-1) !}\left[\sum_{i=0}^{M-m}\left(\begin{array}{c}
N \\
i
\end{array}\right)(\Gamma z)^{i}(1+\Gamma z)^{-N}\right]
$$

Hence, the exact outage probabilities are available from (9) and the exact density from (10)-(13). Hence, the approximate results in [13] can be made exact.

\section{BER CALCULATIONS}

In [13] the BER at the output of the receiver is given by

$$
P_{e}=\int_{0}^{\infty} P_{e}(z) f(z) d z
$$

where $f(z)$ is the SINR density and $P_{e}(z)$ is the conditional probability of error for a given modulation. From [13], [18] we have the conditional probabilities for three binary modulations

$$
\begin{array}{ll}
\text { 1) BPSK: } & P_{e}(z)=\frac{1}{2} \operatorname{erfc}(\sqrt{z}) \\
\text { 2) DPSK : } & P_{e}(z)=\frac{1}{2} e^{-z} \\
\text { 3) OFSK : } & P_{e}(z)=\frac{1}{2} \operatorname{erfc}(\sqrt{z / 2})
\end{array}
$$

where $\operatorname{erfc}(x)=(2 / \sqrt{\pi}) \int_{x}^{+\infty} e^{-t^{2}} d t$ is the complementary error function.

In fact, it usually turns out to be easier to integrate (14) by parts since $F(z)$ or $R(z)$ are more compact than $f(z)$. This gives

$$
P_{e}=\frac{1}{2}+\int_{0}^{\infty} P_{e}^{\prime}(z) R(z) d z
$$

\section{A. DPSK Modulation}

Here, we have the version of (15) given by

$$
P_{e}=\frac{1}{2}\left(1-\int_{0}^{\infty} \exp (-z) R(z) d z\right)
$$

For the general case this was evaluated in [14] giving

$$
\begin{aligned}
P_{e}= & \frac{1}{2}\left(1-\sum_{m=1}^{M} \sum_{i=1}^{N} \frac{\alpha_{i}(m)}{(m-1) ! \Gamma_{i}^{2} \gamma^{m-1}}\right. \\
& \times\left\{(-1)^{m-1} \Gamma_{i}^{-(m-1)} \exp \left(\delta / \Gamma_{i}\right) E_{1}\left(\delta / \Gamma_{i}\right)\right. \\
& \left.\left.+\sum_{k=1}^{m-1}(k-1) !\left(-1 / \Gamma_{i}\right)^{m-1-k} \delta^{-k}\right\}\right)
\end{aligned}
$$

where $\delta=1+1 / \gamma$ and $E_{1}(x)$ is the exponential integral $E_{1}(x)=\int_{x}^{\infty} \exp (-t) / t d t, x>0$. The coefficients $\alpha_{i}(m)$ are given by (5) except that $M$ is replaced by $M-m+1$ in the formula.

For the interference limited system the result is simpler [15] and is given by

$$
P_{e}=\frac{1}{2} \sum_{i=1}^{N} \alpha_{i} \Gamma_{i}^{-2}\left(\Gamma_{i}-\exp \left(1 / \Gamma_{i}\right)\right) E_{1}\left(1 / \Gamma_{i}\right)
$$


For the equal power interference special case substituting (9) in (16) gives

$$
\begin{aligned}
P_{e}= & \frac{1}{2}-\frac{1}{2} \sum_{m=1}^{B} \frac{\gamma^{-(m-1)}}{(m-1) !} \int_{0}^{\infty} z^{m-1} \exp (-\delta z) d z \\
& -\frac{1}{2} \sum_{m=B+1}^{M} \frac{\gamma^{-(m-1)}}{(m-1) !} \sum_{i=0}^{M-m}\left(\begin{array}{c}
N \\
i
\end{array}\right) \Gamma^{i} \\
& \times \int_{0}^{\infty} z^{i+m-1} \exp (-\delta z)(1+\Gamma z)^{-N} d z
\end{aligned}
$$

where $\delta=1+1 / \gamma$. The first integral in (9) is simply the gamma function and the second is also a standard integral [19]. Hence we can write

$$
\begin{aligned}
P_{e}= & \frac{1}{2}-\frac{1}{2}\left(1-(1+\gamma)^{-B}\right)-\frac{1}{2} \sum_{m=B+1}^{M} \frac{\gamma^{-(m-1)}}{(m-1) !} \\
& \times \sum_{i=0}^{M-m}\left(\begin{array}{c}
N \\
i
\end{array}\right) \Gamma^{i} I(i+m-1, \delta, \Gamma, N)
\end{aligned}
$$

and using results [2, eq. (9.210)], [4, eq. (9.211)], and [19] the integral in (20) can be written as

$$
\begin{aligned}
I(r, \delta, \Gamma, N) & \\
= & \frac{\Gamma(r+1)}{\Gamma^{r+1}}\left\{\frac{\Gamma(N-r+1)}{\Gamma(N)} \Phi(r+1, r+2-N ; \delta / \Gamma)\right. \\
& \left.+\frac{\Gamma(r+1-N)}{\Gamma(r+1)}\left(\frac{\delta}{\Gamma}\right)^{N-r-1} \Phi(N, N-r ; \delta / \Gamma)\right\}
\end{aligned}
$$

where $\Phi(\cdot)$ is the confluent hypergeometric function. Computation of $\Phi(\cdot)$ can be achieved through various series methods [20], [21].

\section{B. BPSK and OFSK Modulations}

Here, we have the version of (15) given by

$$
P_{e}=\frac{1}{2}-\frac{1}{2} \int_{0}^{\infty} \operatorname{erf}^{\prime}(\sqrt{\mu z}) R(z) d z
$$

using $\operatorname{erf}(x)=1-\operatorname{erfc}(x)$ and $\mu=1$ (BPSK), $\mu=2$ (OFSK). Differentiating the $\operatorname{erf}(\cdot)$ function in (22) gives

$$
P_{e}=\frac{1}{2}-\frac{1}{2} \sqrt{\frac{\mu}{\pi}} \int_{0}^{\infty} z^{-1 / 2} \exp (-\mu z) R(z) d z .
$$

In [14] the reliability in (1) was expanded in the form

$$
R(z)=\exp (-z / \gamma) \sum_{m=1}^{M} \frac{(z / \gamma)^{m-1}}{(m-1) !} \sum_{i=1}^{N}\left(\alpha_{i}(m) / \Gamma_{i}\right)\left(1+\Gamma_{i} z\right)^{-1}
$$

where $\alpha_{i}(m)$ is defined in Section III-A above. Substituting (24) in (23) gives

$$
P_{e}=\frac{1}{2}-\frac{1}{2} \sqrt{\frac{\mu}{\pi}} \sum_{m=1}^{M} \frac{\gamma^{-(m-1)}}{(m-1) !} \sum_{i=1}^{N}\left(\alpha_{i}(m) / \Gamma_{i}\right)
$$

$$
\times \int_{0}^{\infty} z^{m-3 / 2}\left(1+\Gamma_{i} z\right)^{-1} \exp \left(-\delta_{1} z\right) d z
$$

where $\delta_{1}=\mu+1 / \gamma$. The integral in (25) can be expressed as a complementary incomplete gamma function using the result [6, eq. (2.1.3)], [22]. Hence

$$
\begin{aligned}
P_{e}= & \frac{1}{2}-\frac{1}{2} \sqrt{\frac{\mu}{\pi}} \sum_{m=1}^{M} \frac{\gamma^{-(m-1)}}{(m-1) !} \\
& \times \sum_{i=1}^{N} \alpha_{i}(m) \Gamma(m-1 / 2) \Gamma_{i}^{-(m+1 / 2)} \\
& \times \exp \left(\delta_{1} / \Gamma_{i}\right) \Gamma\left(-m+3 / 2, \delta_{1} / \Gamma_{i}\right)
\end{aligned}
$$

where $\Gamma(v, x)=\int_{x}^{\infty} t^{v-1} \exp (-t) d t$

For the interference limited case $\left(\sigma^{2}=0\right)$ the density given in (4) has a simple form and so the BER formula (14) may be used directly giving

$$
\begin{aligned}
P_{e} & =\sum_{i=1}^{N}\left(\alpha_{i} / 2\right) \int_{0}^{\infty} \operatorname{erfc}(\sqrt{\mu z})\left(1+\Gamma_{i} z\right)^{-2} d z \\
& =\sum_{i=1}^{N}\left(\alpha_{i} / \sqrt{\pi}\right) \int_{0}^{\infty} \int_{\sqrt{\mu z}}^{\infty} \exp \left(-t^{2}\right)\left(1+\Gamma_{i} z\right)^{-2} d t d z \\
& =\sum_{i=1}^{N}\left(\alpha_{i} / \sqrt{\pi}\right) \int_{0}^{\infty} \int_{0}^{t^{2} / \mu}\left(1+\Gamma_{i} z\right)^{-2} d z \exp \left(-t^{2}\right) d t \\
& =\sum_{i=1}^{N}\left(\alpha_{i} / \sqrt{\pi}\right) \int_{0}^{\infty} \exp \left(-t^{2}\right)\left[1-\left(1+\Gamma_{i} t^{2} / \mu\right)^{-1}\right] / \Gamma_{i} d t \\
& =\sum_{i=1}^{N} \frac{\alpha_{i}}{2 \Gamma_{i}}\left(1-\sqrt{\frac{\mu \pi}{\Gamma_{i}}} \exp \left(\mu / \Gamma_{i}\right) \operatorname{erfc}\left(\sqrt{\mu / \Gamma_{i}}\right)\right)
\end{aligned}
$$

using the result [21, eq.(7.4.11)].

For the equal power interferers special case we substitute (9) in (23) to give

$$
\begin{aligned}
P_{e}= & \frac{1}{2}-\frac{1}{2} \sqrt{\frac{\mu}{\pi}}\left\{\sum_{m=1}^{B} \frac{\gamma^{-(m-1)}}{(m-1) !} \int_{0}^{\infty} z^{m-3 / 2} \exp \left(-\delta_{1} z\right) d z\right. \\
& +\sum_{m=B+1}^{M} \frac{\gamma^{-(m-1)}}{(m-1) !} \sum_{i=0}^{M-m}\left(\begin{array}{c}
N \\
i
\end{array}\right) \Gamma^{i} \\
& \left.\times \int_{0}^{\infty} z^{m+i-3 / 2}(1+\Gamma z)^{-N} \exp \left(-\delta_{1} z\right) d z\right\}
\end{aligned}
$$

where $\delta_{1}=\mu+1 / \gamma$. As before in (19) the first integral in (28) is a gamma function and the second can be written as a standard integral. Hence, we have

$P_{e}=\frac{1}{2}-\frac{1}{2} \sqrt{\frac{\mu}{\pi}}\left\{\sum_{m=1}^{B} \frac{\gamma^{-(m-1)} \Gamma(m-1 / 2)}{(m-1) ! \delta_{1}^{m-1 / 2}}\right.$ 


$$
\left.+\sum_{m=B+1}^{M} \frac{\gamma^{-(m-1)}}{(m-1) !} \sum_{i=0}^{M-m}\left(\begin{array}{c}
N \\
i
\end{array}\right) \Gamma^{i} I\left(m+i-3 / 2, \delta_{1}, \Gamma, N\right)\right\}
$$

where $I($.$) is defined in (21).$

\section{CONCLUSION}

We have given an overview of recent results on outage probabilities and bit-error rates (BER) for optimal combiners in the presence of arbitrary numbers of Rayleigh fading cochannel interferers and additive noise. In addition to summarizing previous works, this letter also derives the exact BER for a wider range of modulations than was previously available. This extends the work on approximate BER performance and single or double interferer results and enables a fast and complete analysis of such systems.

\section{REFERENCES}

[1] J. H. Winters, "Optimum combining in digital mobile radio with cochannel interference," IEEE J. Sel. Areas Commun., vol. SAC-2, no. 4, pp. 528-539, Jul. 1984.

[2] J. H. Winters, "On the capacity of radio communication systems with diversity in a Rayleigh fading environment," IEEE J. Sel. Areas Commun., vol. SAC-5, no. 5, pp. 871-878, Jun. 1987.

[3] J. H. Winters, "Optimum combining for indoor radio systems with multiple users," IEEE Trans. Commun., vol. COM-35, no. 11, pp. 1222-1230, Nov. 1987.

[4] J. H. Winters, J. Salz, and R. D. Gitlin, "The impact of antenna diversity on the capacity of wireless communication sysatems," IEEE Trans. Commun., vol. 42, no. 234, pp. 1740-1751, Feb.-Apr. 1994.

[5] J. H. Winters and J. Salz, "Upper bounds on the bit error rate of optimum combining in wireless systems," Proc. IEEE Veh. Technol. Conf., Stockholm, Sweden, pp. 942-946, Jun. 1994.

[6] P. Balaban and J. Salz, "Optimum diversity combining and equalization in digital data transmission with applications to cellular mobile radioPart I: Theoretical considerations," IEEE Trans. Commun., vol. 40, no. 5, pp. 885-894, May 1992.

[7] M. V. Clark, L. J. Greenstein, W. K. Kennedy, and M. Shafi, "MMSE diversity combining for wide-band digital cellular radio," IEEE Trans. Commun., vol. 40, no. 6, pp. 1128-1135, Jun. 1992.
[8] M. V. Clark, L. J. Greenstein, W. K. Kennedy, and M. Shafi, "Optimum linear diversity receivers for mobile communications," IEEE Trans. Veh. Technol., vol. 43, no. 1, pp. 47-56, Feb. 1994.

[9] G. J. Foschini and L. J. Salz, "Digital communications over fading radio channels," Bell Syst. Tech. J., vol. 62, pp. 429-456, Feb. 1983.

[10] H. Gao and P. J. Smith, "Exact SINR calculations for optimum linear combining in wireless systems," PEIS, vol. 12, pp. 261-281, 1998.

[11] H. Gao, "The design and analysis of some computationally intensive communication systems," Ph.D. dissertation, Inst. Stat. Oper. Res., Victoria Univ. of Wellington, Wellington, NZ, Jun. 1996.

[12] H. Gao, P. J. Smith, and M. V. Clark, "Theoretical reliability of MMSE linear diversity combining in Rayleigh fading, additive interference channels," IEEE Trans. Commun., vol. COM-46, no. 5, pp. 666-672, May 1998.

[13] E. Villier, "Performance analysis of optimum combining wiith multiple interferers in flat Rayleigh fading," IEEE Trans. Commun., vol. 47, no. 10, pp. 1503-1510, Oct. 1999.

[14] P. J. Smith and H. Gao, "The polynomial ratio distribution," Research Report No. 99-15, School of Mathematical and Computing Sciences, Victoria Univ. of Wellington. Wellington, NZ, 1999.

[15] P. J. Smith, H. Gao, and M. V. Clark, "Performance bounds for MMSE linear macrodiversity combining in Rayleigh fading, additive interference channels," submitted to the Journal of Communications and Networks.

[16] A. Shah, A. M. Haimovich, M. K. Simon, and M. S. Alouini, "Exact biterror probability for optimal combining with a Rayleigh fading Gaussian cochannel interferer," IEEE Trans. Commun., vol. 48, no. 6, pp. 908-912, Jun. 2000.

[17] V. A. Aalo and J. Zhang, "Performance of antenna systems with optimum combining in a Rayleigh fading environment," IEEE Commun. Lett., vol. 4, no. 12, pp. 387-389, Dec. 2000.

[18] J. G. Proakis, Digital Communications, 2nd ed. New York: McGrawHill, 1989.

[19] I. S. Gradshteyn and I. M. Ryzhik, Table of Integrals, Series, and Products. 5th ed. New York: Academic, 1994.

[20] A. Annamalei, C. Tellambura, and Vijay K. Bhargava, "Exact evaluation of maximal ratio and equal-gain diversity receivers for $M$-ary QAM on Nakagami fading channels," IEEE Trans. Commun., vol. 47, no. 9, pp. 1335-1344, Sep. 1999.

[21] I. Stegun and M. Abramowitz, Handbook of Mathematical Functions with Formulas, Graphs, and Mathematical Tables. Washington, DC: National Bureau of Standards, U.S. Government Printing Office, 1972.

[22] A. P. Prudnikov, Y. A. Brychkov, and O. I. Marichev, Integrals and Series: Direct Laplace Transforms, vol. 4, New York: Gordon and Breach Science, 1986. 